\title{
Une représentation plastique du temps
}

A Plastic Representation of Time (Bwaba, Burkina Faso)

\section{Michèle Coquet et Luc Régis}

\section{OpenEdition}

Journals

Édition électronique

URL : http://journals.openedition.org/span/595

DOl : 10.4000/span.595

ISSN : 2268-1558

\section{Éditeur}

École pratique des hautes études. Sciences humaines

\section{Édition imprimée}

Date de publication : 1 septembre 1986

Pagination : 121-131

ISSN : 0294-7080

\section{Référence électronique}

Michèle Coquet et Luc Régis, « Une représentation plastique du temps », Systèmes de pensée en Afrique noire [En ligne], 7 | 1986, mis en ligne le 05 juin 2013, consulté le 19 avril 2019. URL : http:// journals.openedition.org/span/595; DOI : 10.4000/span.595 


\title{
UNE REPRESENTATION PLASTIQUE DU TEMPS
}

\author{
par \\ Michèle Coquet \& Luc Régis
}

Le savoir concernant la mesure du temps n'est partagé que par quelques individus dans la société bwanu. Si l'alternance du jour et de la nuit, la course du soleil, les phases de la lune, sont des phénomènes observables par tous et appartiennent aux données du temps vécu et à ses qualités sensibles, leur mâ̂trise selon un mode de pensée abstrait et symbolisant relève de la compétence des forgerons. Détenteurs du savoir religieux et organisateurs de la vie rituelle de la communauté (1), ce sont les forgerons en effet qui sont amenés à utiliser un comput calendaire, en procédant à un découpage du temps qui, bien que reposant sur des observations astronomiques, est interprété au travers d'une grille de lecture particulière, celle de la pensée mythique.

Ce savoir est ésotérique : son enseignement est réservé aux adultes ayant généralement atteint 1 a cinquantaine, et sa divulgation serait punie de mort. L'apprentissage a par ailleurs lieu en brousse, sur la montagne du sein de laquelle est extrait le minerai de fer. On voit alors combien la maîtrise que les forgerons exercent en ce domaine est grande, puisque, indépendamment des rites spécifiques à chaque $1 i-$ gnage forgeron ou paysan, ce sont eux qui organisent la vie rituelle communautaire en calculant les périodes et les jours favorables au bon déroulement des cérémonies (2).

Systèmes de Pensée, Cahier 7, 1984 
Si les règles calendaires et le discours mythique qui les interprète sont des données ésotériques, en revanche, 1'expression graphique du temps ou d'un rythme temporel occupe une large placedans 1'univers plastique des Bwaba, non seulement celui des objets, mais également celui des corps à travers les scarifications, puisqu'au comptage des jours et des mois va parfois correspondre un comptage des traits incisés dans la peau. A côté de la représentation figurative de la lune en quartier (en haut des masques en bois, d'origine étrangère, dont la symbolique graphique semble avoir été réinterprétée et redessinée par les Bwaba), il existe un ensemble de signes abstraits exprimant les données d'une mesure temporelle.

I. LA MYTHOLOGIE DES ASTRES

Nous nous attacherons en premier lieu à comprendre comment 1 'interprétation mythique pénètre le comput calendaire et ce qui en est ensuite transcrit sur le plan graphique. Nous ne parlerons donc que très peu de l'organisation des rites en fonction de ce calendrier, en bien des points encore obscur, pour tenter de n'en définir que la structure organisatrice.

Les Bwaba utilisent pour exprimer leur compréhension de cette dimension si difficilement définissable du temps et de sa durée trois concepts fondamentaux, qui semblent organiser en général leur perception du monde et des formes du monde: sanaa, jaho, wiini. Dans 1 'univers des formes, sanaa - corps désignerait la substance des choses, leur matière, jaho-visage leur surface apparente sur laquelle viennent s'inscrire les wiini, de wi, inciser, graver, terme employé pour nommer les scarifications, et tout ce qui apparaît come une trace, sur un plan ou dans 1 'espace, faisant intervenir dans le continu du monde l'idée d'une discontinuité. Replacés dans le discours cosmologique, le sanaa est le Soleil (wii, terme désignant I'astre solaire) le jaho est la Lune (piihun, "1a lune"), et les wiini sont les jours (wizzonze).

La genèse du monde a comme acteurs principaux et originels le Soleil et la Lune, le sanaa et le jaho. De ce premier couple céleste, naissent la terre, les hommes et surtout, avant 1'apparition dupremier 
être humain, des créatures surnaturelles; cet ensemble forme une base numérique stable qui vient expliciter la comptabilité calendaire.

Le soleil était seul avant la création du monde. Désirant avoir une compagne, il fabriqua la Lune, han piihun, "Femme-Lune", avec la terre dont il était fait, et de l'eau. Le Soleil et la Lune restèrent "collés" ensemble (référence à 1 'accouplement céleste) tandis que 1 'obscurité régnait. Le Soleil créa ensuite deux êtres invisibles et androgynes, toujours à partir de son propre corps, la terre : doo (appelé aussi wii tienro, "1'ombre du soleil") (3), et sa jumelle handoo (dite aussi piinun tienro" "1 'onbre de la Lune"); doo est un "homme à seins", jenbaa, mais de sexe masculin, et handoo, bien que de sexe féminin, est barbue et ne possède qu'un seul sein. Ces jumeaux sont cependant conçus comme étant au nombre de trois. Le troisième se nomme binluio : tout d'abord enfant conçu en rêve par doo et handoo, il devient comme ceux-ci oeuvre de terre du Soleil. Il est bicéphale et bisexué, de sexe masculin dans sa partie gauche et féminin dans sa partie droite. Ces deux parties sont considérées comme jumelles, car "1'homme est jumeau, henbeni, dans son corps", de telle sorte que le jumeau mâle est appelé douro et son homologue féminin hankoro, "mauvaise femme".

Suite à la création de ces cinq premiers êtres, vient celle du monde, demandée par han piinum, la Femme-Lune, où s'installent le couple céleste et ses descendants. Ce monde est archaique, ne connaît pas encore 1'alternance du jour et de la nuit, et chacun s'y nourrit de cueillette, des fruits des arbres, dans une errance désordonnée. Une faute est alors commise par hankoro qui tue son père, doo, geste qui va marquer l'apparition de la mort sur terre. Pour racheter la faute, handoo, appelée dans cet épisode de sa vie terrestre maani pon, "main gauche fermée", en référence à son rôle d'initiatrice auprès du genre humain, est sacrifiée avec son fils et "jumeau" bintuio, enterrés tous les deux vivants sous le bois de l'enclume sacrée de la première forge. Cet évènement marque l'entrée dans l'ère terrestre, celle des hommes désormais mortels et tenus de peiner pour se nourrir, et la séparation irrémédiable d'avec le monde surnaturel et divin : en effet, à partir de ce moment-1à, le Soleil et 1a Lune se sont séparés de la terre et "décollés" dans le ciel, l'un engendrant le cycledes jours et des années, I'autre celui des nuits et des mois.

Les cinq êtres surnature1s, doo, handoo, binluio, douro et

hankoro sont appelés wiini du Soleil et de la Lune :

"Les wiini sont les enfants du sanaa et du jaho, ils sont jahoza"

("enfants de jaho").

Ils sont par ailleurs dissociés dans leurs fonctions, ayant chacun un rôle spécifique et donnant chacun un nom à un jour de la semaine, cette semaine de cinq jours, qui apparaît alors comme le fruit de l'accouplement du Soleil et de la Lune.

Si les cinq jours sont chacun fils ou fille du couple céleste,

* Ce récit restitue une mythologie qui nous a été donné par fragments. 
i.1s sont aussi, dans 1 eur succession et leur éternel recommencement, 1a manifestation de la course perpétuelle du soleil derrière la lune ("le jaho est toujours devant le sanaa et le sana ne peut pas marcher sans le jaho"), leur rencontre ne se produisant qu'épisodiquement lors de l'éclipse de soleil, wii wan piihun, "le Soleil a avalé la Lune"(4). Le mythe dit:

"Les jours sont comme une femme et son mari. C'est une rencontre car c'est le sanaa et le jaho qui ont donné la semaine de cinq jours pour se rencontrer".

En effet, les cinq jours sont les enfants du sanaa et du jah̆o, et sont appréhendés comme une succession où 1'un engendre l'autre, indéfiniment. Chaque jour est done gardé en quelque sorte, ou confié, karafa, à la divinité dont il porte le nom. Chacune porte plusieurs noms selon les rôles qu'elle joue dans un contexte narratif précis. Ainsi se succèdent:

karafahan, "femme confiée", jour de handoo : elle commande l'eau du marigot. Lorsque celui-ci s'assèche de façon imprévisible, c'est que des fautes ont été commises au village.

karafako, jour de doo : il commande le feu, et en particulier les feux de brousse. Mais c'est karafahan qui ordonne de mettre le feu à la brousse.

karafaza, "enfant confié", jour de binluio, qui commande aux abeilles. Mais c'est karafako qui lui ordonne de faire s'envoler l'essaim pour punir les hommes de leurs fautes.

douro, est le gardien de la brousse. Mais c'est karafaza qui donne les ordres : ne pas faire tomber la pluie en hivernage s'il y a eu accouplement en brousse, ou perdre le chasseur qui n'a pas respecté les règles.

hankoro, "mauvaise femme", est celle qui détourne les esprits et apporte la discorde entre les humains.

Ce mode parabolique d'expression de la production des jours les uns par les autres révèle combien est grande l'interdépendance entre chaque jour, considéré comme une unité temporelle prise dans une succession. Chaque jour/unité devient ainsi le maillon d'une chaîne et se trouve en corrélation avec le jour passé et le jour à venir.

La disjonction originelle du Soleil et de la Lune d'avec la terre provoque l'alternance du jour et de la nuit, des saisons, saison des pluies et saison sèche, l'organisation des mois et des jours. Cependant, ce phénomène conjonction/disjonction se renouvelle chaque jour, lorsque le soleil se couche (conjonction avec la terre), lorsqu'il se lève à 
nouveau (disjonction). Selon la mythologie forgeronne, le soleil conjoint à la terre est localisé sous le bois de l'enclume sacrée, au centre de la forge.

La lune également, selon des phases temporelles plus espacées, c'est à dire lors de sa période descendante et lorsqu'elle n'est plus visible, se conjoint à $l^{\prime}$ eau du marigot sacré, nioun dani, lieu où résident les enfants à naître.

En conclusion, nous dirons que le temps, à l'image de toute chose organisée, est constitué d'un sanaa, d'unjaho et de wiini, et qu'à ce titre, si $1^{\prime}$ on se réfère à la pensée bwanu, le temps est un être vivant, résultat d'un engendrement (exprimé par le terme wiini et représenté par les cinq descendants du couple céleste et la course du Soleil après la Lune), et se définit également comme un processus de production (succession des jours) (5).

\section{II . LES RYTHMES TEMPORELS ET LEUR REPRESENTATION}

Les rythmes temporels qui composent le temps sensible, indépendamment des faits astronomiques, sont transcrits graphiquement et formellement. Aux différents rythmes de la réalité objective vont correspondre autant de signifiants plastiques. En repartant des structures mythiques, nous nous référerons aux ordres temporels fondamentaux, celui du soleil et celui de la Lune, principes actifs et organisateurs, puis aux unités de temps, mesurées et comptées, qui en découlent.

Le temps sotaire

L'astre solaire organise le temps de la journée, wiizonze, tandis que la nuit est régie par l'ordre lunaire. L'apparition du soleil à 1 'aube se dit wii ha, le soleil s'est levé, puis wii bon, le soleil est monté (1itt. "soleil cuit", de be, bon, cuire), wii zon mi kahun, le soleil est rentré dans son trou, à 1 'instant du zénith, puiswii jenie, "soleil incliné", interprété également comme le soleil s'étant déplacé dans son trou, et enfin wii to, "le soleil est tombé".

La journée est bipartite : 1a période s'étendant du lever au début 
du déclin est appelée sanaa. Arrivé au zénith, le soleil est au point culminant de sa course, et la seconde partie de la journée est nommée jaho, comme si on évitait de parler de la phase déclinante de l'astre. Cette période est pensée comme faisant partie du lendemain. En fonction de cette bipartition s'organise la vie rituelle : ainsi, les sacrifices à la forge, à l'enclume sacrée, aux ancêtres du lignage, doivent s'effectuer avant $1^{\prime}$ amorce du déclin du soleil. Par contre, un grand nombre de cérémonies de masques ont lieu 1 'après-midi et durant le crépuscule. Le jaho est alors non seulement la lune, mais également. la référence à l'obscurité à venir et le fait que le temps de I'aprèsmidi soit jaho, ce qui est "toujours devant le sanaa", marque bien cette volonté d'exprimer l'orientation de l'écoulement du temps.

La position zénithale du soleil est également appelée wii kotin, "le soleil est au coeur", c'est à dire au centre. Les expressions "le soleil est rentré dans son trou", puis "le soleil s'est déplacé dans son trou" font référence au moment où 1'être mythique sanaa-Soleil retourne, après sa course dans le ciel, sous le bois de l'enclume sacrée. Pourtant, cette formule désigne l'instant où l'astre est au sommet de sa course. Il semblerait que ce soit en relation avec cette non-considération de la phase déclinante, appréhendée peut-être comme dysphorique pour préfigurer la conjonction du soleil et de la terre et revenir ainsi à l'obscurité primordiale. Quoi qu'il en soit, la journée solaire est envisagée comme une et indivisible et se répétant à l'identique chaque jour. Le temps solaire s'inscrit dans un continu, sur un mode duratif, où I'homme se trouve pris dans un temps éternel, qui est le temps physique, linéaire et infini, également le temps de la matière, celle-là même (la terre) dont sont faits le sanaa, les premiers humains, et à laquelle ceux-ci retournent à leur mort : le sanaa, disent les Bwaba, est "le corps de toutes les choses".

Deux signes graphiques, la spirale et la figure du rayonnement, servent à signifier l'astre solaire et exprimer l'ordre temporel qui lui est corrélé. On les trouve sur des médaillons en bronze, appelés wii, portés par les enfants mâles que l'on dit mourir et renâtre successivement. Pour arrêter la répétition du cycle, on procède, entre autres rituels, à la fabrication du pendentif (6). Les motifs spiralés ou rayonnants que l'on trouve sur ces objets ont atteint une grande perfection géométrique ( $\mathrm{fig} .1,3,4)$. On les rencontre également 
sur d'autres supports, comme les instruments métalliques : manches spiralés de couteaux de sacrifice, bracelets torsadés, lames de scarifications, briquets traditionnels (fig. 2). La volute spiralée qui termine ces outils est nommée "queue du caméléon". Le motif rayonnant, quant à 1ui, est inscrit plus généralement sur les corps, autour du nombril, kuiare, ou kuiotienhun, "le milieu du corps". Cette scarification est considérée comme la première à avoir été révélée; le nombril est le lieu où se trouve l'âme boni et est comme le centre de la terre $(7)$.

Nous ne nous attarderons pas sur le symbolisme de la spirale (connu de la tradition mythique et religieuse); rappelons simplement ses propriétés signifiantes de structure ouverte, tendant au non-fini : le rythme spiralé, de même que le rayonnant, obéit à une logique interne de développement temporel s'inscrivant dans une spatialité en expansion. La spirale en tant que courbe tournant autour d'un pôle et le rayonnement, organisé $1 u i$ aussi autour d'un point, marquent 1'importance du centre, appelé là aussi Za kotinn, "centre de la terre". Au verso du médailion (fig. 3) se trouve une croix, motif également présent dans 1 a structure rayonnante. Cette croix est la représentation du premier forgeron créé par le sanaa, et indique également les directions du 1evant et du couchant. Nous avons ainsi, sur un même objet, 1'idée du temps dilaté, qui est le temps solaire, transcrit spatialement par les motifs rayonnants et spiralés, et une définition de l'homme comme situé au centre, observateur idéa1 de la course du soleil, d'Est en Ouest.

Le temps Zunaire

Le temps du jaho débute dès l'instant où le soleil commence à décliner, jusqu'à 1 'aube. On observe que du déclin jusqu'au crépuscule, le temps solaire et le temps lunaire "s 'interfèrent".

Comme ceux de la journée, les moments de la nuit sont nommés : Za junbikaa, le crépuscule, Za hun, il fait nuit, ou tinanhinn, "jour obscurci", de hi, hun, épaissir, devenir compact, employé entre autre pour désigner un certain état de la potasse ou du beurre durant leur préparation, puis zo van ju, la nuit s'est avancée. Za nè marque le 
milieu de la nuit que les Bwaba situent vers une heure du matin, de $n i$, nè, "éclater", qui est le même verbe utilisé pour signifier la détonation du fusil. Ensuite, les chants des coqs vont annoncer l'aube, celui du kojaba, le premier coq, puis mandi kobia, le "coq du dos", c'est à dire le dernier coq: Za tan est l'aube, de ti, griller, brûler, ou Za bara, le jour s'est déplié (comme on le dit d'un tissu).

Cette liste n'est bien sûr pas exhaustive et d'autres expressions permettent sans doute de définir les moments de la nuit. Cependant, elle nous amène à noter la présence de la métaphore de la cuisson, relevée déjà dans wii bon, "soleil cuit" (8).

Les Bwaba ne semblent prendre en compte que la phase ascendante de la lune, jusqu'à ce qu'elle soit pleine. Le mois, piikere, "lune unique", est de quinze jours, les quinze autres jours de la phase descendante n'étant pas comptabilisés. La phase dócrỗssante est appelée pinbii, "1une noire", ou piinun tibiri, "lune obscurité". L'année, zuzure, compte les sept mois ou les sept lunes du temps de l'hivernage (dès 1 'apparition des premières pluies); le septième mois serait le mois d'entrée en saison sèche. Celle-ci, non comptabilisée, est comprise comme 1 'intervalle entre deux saisons des pluies, venant préparer par ses manifestations religieuses le bon déroulement des activités agricoles de la saison des pluies, moment où han piihun, "Femme-lune", divinité de l'eau, (se rappeler son séjour dans le marigot) fait tomber la pluie. De cellemci dépendent donc la fécondité des sols comme celle des femmes. C'est à elle que sacrifie le la Banso lors des sécheresses (ou des manques d'enfants).

Les phases de la lune sont définies en fonction de la position de 1 'astre dans le ciel par rapport au lever et au coucher du solei1. La phase ascendante est pin finze, "lune neuve", piimun man, "la lune est vue", lorsqu'elle apparâ̂t à 1'ouest en quartier très mince, ou pinza, "lune enfant"; 1a pleine lune est ka kirikipi, "comme le cercle", ou piikoomu, "en pleine lumière", comme on dit du champ dégagé des hautes herbes et sarclé, ou piidau, "1a lune a fini de crôtre", comme le mil lorsqu'il est arrivé à maturité et est prêt à être récolté, ou encore piihun zon mi kahun, "la lune est rentrée dans son trou": Le même phénomène semble alors se produire que lors du cycle solaire : 1 'astre lunaire, au quinzième jour, est arrivé à son développement maximal et sa disparition prochaine est anticipée à travers ses dénominations. Ce moment arrivé, les Bwaba considèrent que "hanpiiłzun a 
fini sa visite au monde". Survient un temps de six jours, associée à la période menstruelle de hanpiihun (9) où la lune est jieniè "'penchée", ou huara, "agenouillée", à l'est, à peine visible au-dessus de l'horizon. Cet "agenouillement" est conçu comme un rituel de "pardon", sabara, destiné à prévenir les maux pouvant arriver avec la disparition de la lune, moment considéré comme le plus dangereux de la phase décroissante. Lorsque l'astre aura disparu à l'est, il sera appelé piinun lu, "Iune immergée", en référence à 1 'époque du mythe où hanpii ihun, 1a FemmeLune, réside dans l'eau du marigot sacré, nioun dani.

La phase ascendante est la période bénéfique où va s'exercer la majeure partie de l'activité rituel1e. C'est à ce moment-1à que les semailles sont faites (la récolte, elle, commencerait au premier jour de la lune décroissante). C'est également la période durant laquelle s'effectuent les mariages, où se pratiquent les consultations divinatoires, où les accouchements qui surviennent sont bénéfiques à l'enfant nouveau-né, où ont lieu les funérailles, où les masques de feuilles à plumes de calao, bieni, sortent, où $1^{\prime}$ on dit les contes, etc.

Par contre, la période de décours de la lune semble vécue comme un moment dangereux. Dès que $1^{\prime}$ astre n'est plus visible, des sacrifices animaux sont exécutés par le chef des forgerons au bord du marigot sacré, et à 1 'autel des ancêtres forgerons. Une mort survenant à ce moment-là pourra dans certains cas ne pas être suivie de funérailles, ou tout du moins nécessitera un certain nombre de rites propitiatoires; de même pour une naissance. Durant cette même période, les rapports sexuels sont interdits à ceux qui ont des charges sacrificielles.

Pour les femmes nées en lune noire - et semble-t-il pour les enfants mâles issus d'un rapport sexuel pratiqué alors que la mère était en période menstruelle -, est fabriqué un pendentif, à vocation propitiatoire, en forme de croissant de lune, dans la position pizinun jienié, "lune penchée", ou pirbii, "lune noire". Ce pendentif (fig.6) est compris comme la réunion de deux parties symétriques, corps de deux caméléons dont les queues spiralées se retrouvent aux deux extrémités de l'objet. Un autre pendentif ( $\mathrm{fig}$. 5), destiné aux enfants nés jumeaux, également en arc de cercle, figure deux caméléons superposés, en position d'accouplement. Le caméléon est un animal très présent dans $1 a$ cosmogonie : si la spirale de sa queue est une représentation du Soleil, sa tâche est de conduire l'astre lunaire tout au long de sa course noc- 
turne; il serait également l'une des nombreuses manifestations de doo. I1 joue un rôle important dans les rituels de pluie et son effigie est utilisée à cette occasion par les devins. Le caméléon est conçu en couple, de nature gémellaire et bisexuée. De la même façon, les deux phases de la lune, pin finle et pinbii, sont pensées par les Bwaba comme deux parties jumelles. Le temps lunaire repose sur un principe d'alternance. Une des manifestations plastiques les plus remarquables en est celle que 1'on trouve sur les couvertures, portées par les hommes les plus vieux du lignage, nunu, en damier noir et blanc, chaque carré noir représentant une phase de la lune décroissante, et chaque carré blanc une phase de la lune croissante (10) (Cf. figure 7). Principe d'alternance organisé également selon une structure synétrique, se développant autour d'un axe. La bipartition du jaho-lune est attestée dans la définition même du jaho: le jaho présuppose le sanaa, comme la "face" présuppose pour exister un "corps", alors que le sanaa, en tant que matière originelle, se suffit à lui-même.

Par ailleurs, la courbe qui relie. les deux extrémités spiralées, donc solaires, du pendentif pinbii (fig.6) contient ce mouvement, cette représentation synthétique de la course de la lune allant du levant au couchant, les incisions sur le croissant marquant la succession des jours nécessaires à $l$ 'astre pour accomplir sa révolution. Ainsi, sur les deux pendentifs (fig. 5\&6), se trouvent non seulement le signe solaire de la spirale, mais également un réseau de hachures, kahalanki wiini, les "scarifications du caméléon", ce qui nous amène à parler de ce module de comptabilité du temps, la semaine de cinq jours, issue de l'observation des deux cycles lunaire et solaire et, comme nous l'avons vu, de l'union du couple céleste.

Les jours de la semaine, wini.

L'élaboration du calendrier religieux se base sur un décompte de jours groupés en une semaine traditionnelle de cinq jours. Celle-ci est ponctuée par un marché coutumier intervenant chaque cinquième jour dans chaque village, et ceci en dépit d'une part des grands marchés mis en place par l'administration coloniale, ayant lieu tous les sept jours dans les villages les plus importants, d'autre part de l'adoption quasi 
générale de la semaine musulmane de sept jours - teniè, haraBa, tarata, Zaamisa, jazuma, tibiri, hati. Le clivage existant entre la semaine traditionnelle et 1 a semaine musulmane entraîne une adaptation de cette dernière à l'ancienne, se manifestant par le respect du module de base de cinq jours et I'adjonction de deux jours "qui sont", mais qu" "on ne compte pas".

Wiini est un terme générique désignant non seulement tout ce qui fait trace, par incision ou gravure, mais aussi la charpente linéaire qui semble structurer tout être vivant, le squelette, les branches d'un arbre ou les nervures d'une feuille, de même que les poteaux de bois qui soutiennent la maison ou les sillons d'un champ de culture. Le temps des winin, celui des jours, une journée comportant une nuit et 1a phase montante du soleil ( 1 'après-midi appartient déjà au jour suivant) est la durée segmentée, qui structure le temps vécu, le traverse et le divise en autant d'unités semblables. Chaque jour de la semaine, toujours recommencé, est cependant individualisé pour être sous la tutelle d'un des cinq êtres surnaturels de la mythologie. Un ensemble de prescriptions et d'interdictions dépendant de ces divinités régissent les actes que 1 'on doit accomplir et organisent ainsi la vie sociale et rituelle de la communauté. En voici quelques exemples:

- le premier jour, jour de handoo, est favorable aux baptêmes et aux mariages.

- le deuxième jour, jour de doo, est également favorable aux baptêmes et aux mariages, mais les sacrifices destinés à 1'autel-grenier construit pour les jumeaux sont interdits, ainsi que de manger du tô de sorgho rouge.

- le troisième jour, jour de birluio : 1'enlèvement des femmes, $1^{\prime} \mathrm{ex}-$ cision et le rituel des scarifications sont prohibés.

- le quatrième jour, jour de douro, est favorable aux sacrifices faits par les devins, au modelage des statuettes divinatoires et aux chasseurs.

- le cinquième jour, jour de hankoro, est un jour néfaste où I'activité rituelle est suspendue.

Ainsi, pour accomplir un rituel sacrificiel important, il faudra tenir compte de cet ensemble de prescriptions pour choisir(par divination) le jour, considérer le moment de la journée (avant le déclin du soleil) et du mois (phase ascendante de la lune). Ces calculs de temps se représentent par des successions linéaires de traits, chaque trait renvoyant à un jour, quinze traits faisant quinze jours, soit un mois. 
I1 apparaît cependant que l'ensemble d'une série de traits effectivement représentés ne corresponde jamais à une somme idéale, comme la somme de quinze qui est signifiante. La formule serait plutôt : dans cet ensemble de traits, il y a les quinze jours du mois, ou bien les sept jours que l'on compte avant de faire tel sacrifice. Il s'agirait alors de la représentation du concept de comptabilité plutôt que de celle d'une comptabilité réelle et arithmétique. Le principe itératif qui régit cette transcription est le même que celui qui structure les signes des scarifications corporelles bwaba.

Pourtant, tous les signes du visage ou du corps ne semblent pas être interprétés comme la représentation de jours, mais seulement certains d'entre eux tel que le signe de la cithare, cienhun, que tous les Bwaba doivent porter et qui se situe sur chaque tempe (fig. 8). "Tous les jours et les mois sont dans la cithare" disent-ils.

Ce signe se compose en principe de deux rubans de quinze traits chacun, sur chacune des deux tempes, soit trente incisions à gauche et trente à droite. Chaque ruban équivaut à un mois et le vide entre les rubans est 1à où siège hanpiithun boni, 1'âme de la Femme-Lune. Leur exécution exige de 1 'opératrice, la femme forgeronne qui fait les scarifications, un comportement singulier qui lui impose de retenir sa respiration 1orsqu'elle coupe la peau tous les trois traits (chiffre de I'homme), puis tous les quatre traits (chiffre de la femme).

Deux autres signes, situés de part et d'autre du front,

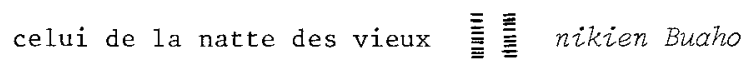
et celui de la natte des accouchements :":": haaba tenni Buaho renvoient eux aussi à une comptabilité de jours. Le premier est destiné aux. enfants mâles (des forgerons et des familles du chef de terre) qui auront à remplir des charges sacrificielles importantes et le second aux futures sages-femmes. Dans les deux cas, il ne s'agit pas, encore une fois, d'une somme de jours correspondant à une somme de traits, mais d'un certain nombre de jours, propre à chaque signe, qui sont ceux que l'on compte avant de sacrifier à l'objet. La même chose se produit pour le signe de la cithare: les trois objets appartiennent en effet à la mythologie lunaire. Ils sont d'ailleurs de structure identique : composés de brins de paille, accolés, pour la cithare, et de baguettes de bambous pour les deux autres, les tiges étant liées 
entre elles par une cordelette de coton et de cuir. Dans le même ordre d'idée, tous les objets construits par addition d'éléments semblables seront des objets "calendaires", ou se référant à l'idée d'une segmentation d'une durée temporelle : ainsi, la succession des plumes blanches (couleur de pinfinte, la "lune neuve") du calao portées en cimier sur certains masques de feuilles (le signe des plumes de calao, bakuun kuzaaho, se retrouvant 1 ui aussi dans les signes du visage). Le calao est par ailleurs une des manifestations de binzuio et était chargé de guider doo, le futur masque de feuilles, dans ces temps originels du mythe où la terre était plongée dans l'obscurité. Ce signe est réservé aux enfants des prêtres du doo, doo Banso et aux enfants de la famille du chef de terre.

Les wiini segmentent en unités égales, en quantité fixes, le continu du temps, et remplissent ainsi cette fonction de charpente qui est la leur.

La subdivision en cinq jours, les activités prescrites durant ces cinq jours d'après les données du mythe, ne changent rien à cette fonction de division d'une durée infinie, traduite graphiquement par un motif linéaire, interrompu de façon régulière, sans centre, à 1 'inverse de la spirale (11).

Nous reprenons pour résumer l'objet pinbii (fig.6) comme exemple de la transcription de ces trois qualités de temps auxquelles se réfèrent les Bwaba :

la spirale le temps sana $\alpha$, qui est un temps non segmenté et continu mais comportant un point de départ (le centre) renvoyant à la création du monde.

la structure le temps jaho, qui est un temps segmenté en unités, les bipartite mois, mais dont I'organisation repose sur un principe d'alternance.

la succession le temps wiini, qui est un temps segmenté en unités plus de traits petites, les jours, mais qui est un temps uniforme, obéissant au principe d'itération.

La succession de traits régulièrement inscrits que sont les wiini semble appartenir à un mode d'expression parmi les plus archa $\mathbf{i}-$ ques. Nous nous référerons ici à $\mathrm{A}$. Leroi-Gourhan (12) qui remarque que ce graphisme est la première trace d'expression que l'on ait trou- 
vé dans 1'histoire des formes et correspond à un rythme primaire, celui de la parole, mais aussi du coeur, de la marche ou du martèlement. Sans aller si loin, nous notons simplement que dans I'univers formel bwanu, la succession de traits est en effet un mode de représentation plus élémentaire que la figure en croissant, la spirale ou le rayonnement qui sont de nature plus complexe. Or cette succession de traits est ce qui compose le système graphique des scarifications, considérées par les Bwaba eux-mêmes comme ce qu'il y a de plus ancien dans leur culture. Que cette itêration ait à voir avec un rythme primaire se manifesterait par deux comportements particuliers, que nous avons déjà notés : 1 'un, lorsque la femme qui fait les scarifications retient son souffle en pratiquant les incisions, marquant ainsi l'importance du rythme respiratoire, 1 'autre, lors du rituel de $1^{\circ}$ 'éclipse, qui consiste en un martèlement régulier d'une pierre contre l'autre, comme si les Bwaba énonçaient ainsi leur désir de revenir au rythme normal de 1a succession des jours et des nuits, interrompu par l'éclipse.

Une dernière remarque concernant le sens investi dans les signes de la spirale et du rayonnement par rapport à 1a succession des traits, d'un point de vue spatial : les deux premiers renvoient à un espace centré qui est également interprété comme le village, tandis que le dernier semble être utilisé pour exprimer l'idée d'un déplacement.

Quoi qu'il en soit, tous les Bwaba ont, inscrit sur le corps en réseau complexe de signes, cet ordre temporel organisateur et régulateur qu'est le calendrier lunaire. La fonction de reconnaissance des scarifications, en particulier le signe de la cithare, des deux nattes et même le signe solaire du nombril, reposerait entre autre sur le fait que les Bwaba se pensent comme ceux qui partagent le même temps et qui obéissent au même calendrier.

Michèle Coquet \& Luc Régis

Groupe de Recherches Sémio-linguistiques U.R.L. 7 - C.N.R.S. 
(1) Nous ferons tout de suite deux remarques :

a) le terme buanu, pl. buaBa, est la dénomination utilisée pour désigner les agriculteurs, les forgerons étant nommés likinu, pl. ZikiBa. Il n'existe pas d'appellation concernant 1 'ensemble de la communauté. Nous transcrivons par bwanu, bwaba et conservons ce terme contne dénomination générique.

b) chez les Bwaba méridionaux de la région de Houndé, les forgerons forment un groupe endogame et chaque 1 ignage de forgeron, dans un village donné, est attaché au service de lignages paysans. A côté du système d'échanges de prestations habituelles d'ordre économique, une partie des récoltes contre la fourniture d'outils agricoles, existe également un échange de services religieux : la forge est I'aire rituelle centrale du village, et les forgerons, les dépositaires des rites et des mythes de la communauté. Toute la connaissance de la cosmogonie leur appartient et les paysans leur reconnaissent cette science, tout en ayant de leur côté des rites et des mythes propres, concernant la terre et le travail de la terre. Cependant, les forgerons conservent là encore une position dominante, d'une part parce qu'ils sont les pourvoyeurs de tous les instruments en métal du rituel, de l'autre parce que le prêtre de doo, religion communautaire, réunissant les buaBa et les $1 \mathrm{iKiBa}$, est presque toujours le chef du lignage forgeron.

(2) Il arrive qu'un lignage forgeron s'éteigne par manque de descendants mâles. Afin que la vie religieuse se maintieme, la communauté peut faire appel à un lignage forgeron d'un village voisin, ou bien à une catégorie particulière d'individus appelés zumBa. Ceux-ci sont issus d'unions adultérines entre forgerons et paysans. Si la mère est buanu, l'enfant reste buanu, mais est appelé à tenir un rôle particulier, celui de chasseur et de guerrier. A cet individu sera confié, en cas de besoin, un certain nombre de connaissances, et en particulier celle de la maîtrise des jours, en attendant qu'un nouveau groupe forgeron vienne s'installer au village.

I1 existe d'ailleurs également le cas d'enfant de mère forgeronne et de père paysan. L'enfant reste alors forgeron, mais on lui reconnâtra ensuite certains comportements spécifiques dus à sa nature double, celui en particulier d'être toujours en mouvement et de n'être jamais à la forge, tout comme les zumBa buaBa, qui ne restent pas au village pour être toujours en brousse.

(3) L'ombre est considérée comme la manifestation visible de 1'âme boni: "1'ombre, c'est cela ton dieu qui doit être toujours à côté de toi".

(4) Lorsqu'un tel évènement survient, les villageois cessent toute activité et les forgerons, afin de séparer les deux astres, frappent deux pierres-marteaux 1 'une contre 1'autre.

(5) Cette fonction d'engendrement et de transformation attribuée à 1a réunion du sanaa et du jaho se retrouve exprimée de façon similaire dans la symbolique de la forge : si le sanaa est la terre, il est également le feu, et la manipulation combinatoire des deux éléments, feu et eau (jaho), permet l'obtention des objets métalliques, du minerai à l'outil, le feu pour le couler et le chauffer, l'eau pour le refroidir et le durcir à la détrempe. 
(6) Le bijou est ensuite gardé par le chef de terre, Za Banso, et serait également utilisé dans certains sacrifices à la terre.

(7) On présentera d'ailleurs le nouveau-né en un lieu géographique nommé la kotin, "centre de la terre", que possède chaque village, avant de lui couper le cordon ombilical.

(8) A l'inverse, lors de la préparation de la sauce cieni, "feuilles de baobab", dans un contexte rituel (accouchements, baptêrnes). les étapes de la cuisson seront comparées aux phases de la lune : la cuisson se partage en deux temps, une phase montante du liquide et une phase descendante. L'eau dans laquelle sont plongées les feuilles de la cueillette est mise à chauffer dans un canari. Lorsqu'elle se met à bouillir jusqu'à menacer de déborder, c'est la phase ascendante. Le volume de liquide porté à son maximum correspond à 1 'apogée du cycle, lorsque la lune se présente comme un disque complet. A ce moment-là, la cuisinière jette quelques pincées de potasse qui suffisent à faire diminuer la préparation, et remue à l'aide d'un fouet, vinKo, le corps de han piihun, "Femme-Lune", afin de lier la sauce; ce geste est accompagné de paroles rituelles de salutation à han piihun. L'action de la potasse fait baisser le liquide et cette phase est comparée à la phase descendante de l'astre.

(9) L'association phase lunaire/cycle menstruel de la femme est très présente dans la pensée bwanu. Cette période de six jours est donnée comme "amenant de mauvaises choses dans l'esprit des femmes".

(10) Le même damier s'observe sur les masques à lames de la même région (fig. 7).

(11) Cette transcription du temps uniforme mais segmenté existe évidemment dans d'autres cultures. Nous en avons trouvé un exemple cité par Patrick Bidou, in "Le chemin du soleil", L'Homme, $\mathrm{n}^{\circ} 93$, janvmars 1985, p. 93. Il existe chez les Indiens Tatuyo un terme kari, dont le sens nous semble assez proche de celui de wiini. kari, dit 1 'auteur, "correspond à une configuration horizontale de l'espace; il relie des éléments de même nature de manière à former un ensemble continu, il abolit le temps en le rendant étale". Kari entre ainsi dans la composition de mots désignant des objets composés d'une succession d'éléments identiques tels que collier de dents d'animal, coiffures de plumes et même les mains unissant les danseurs les uns aux autres.

(12) A. Leroi-Gourhan, in Le Geste et la Parole, La mémoire et les rythmes, Albin Michel, 1965, Paris, p. 142. 

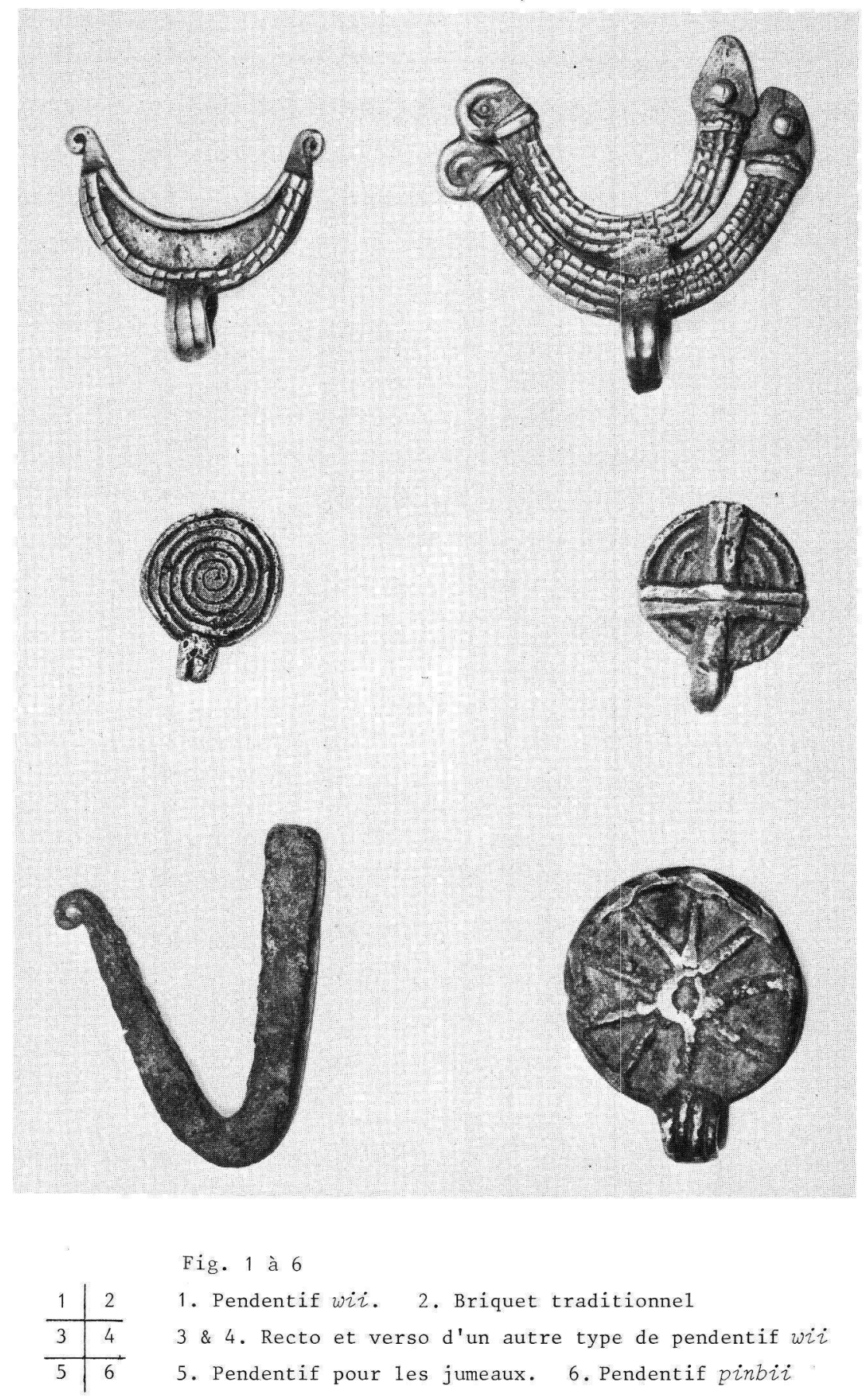


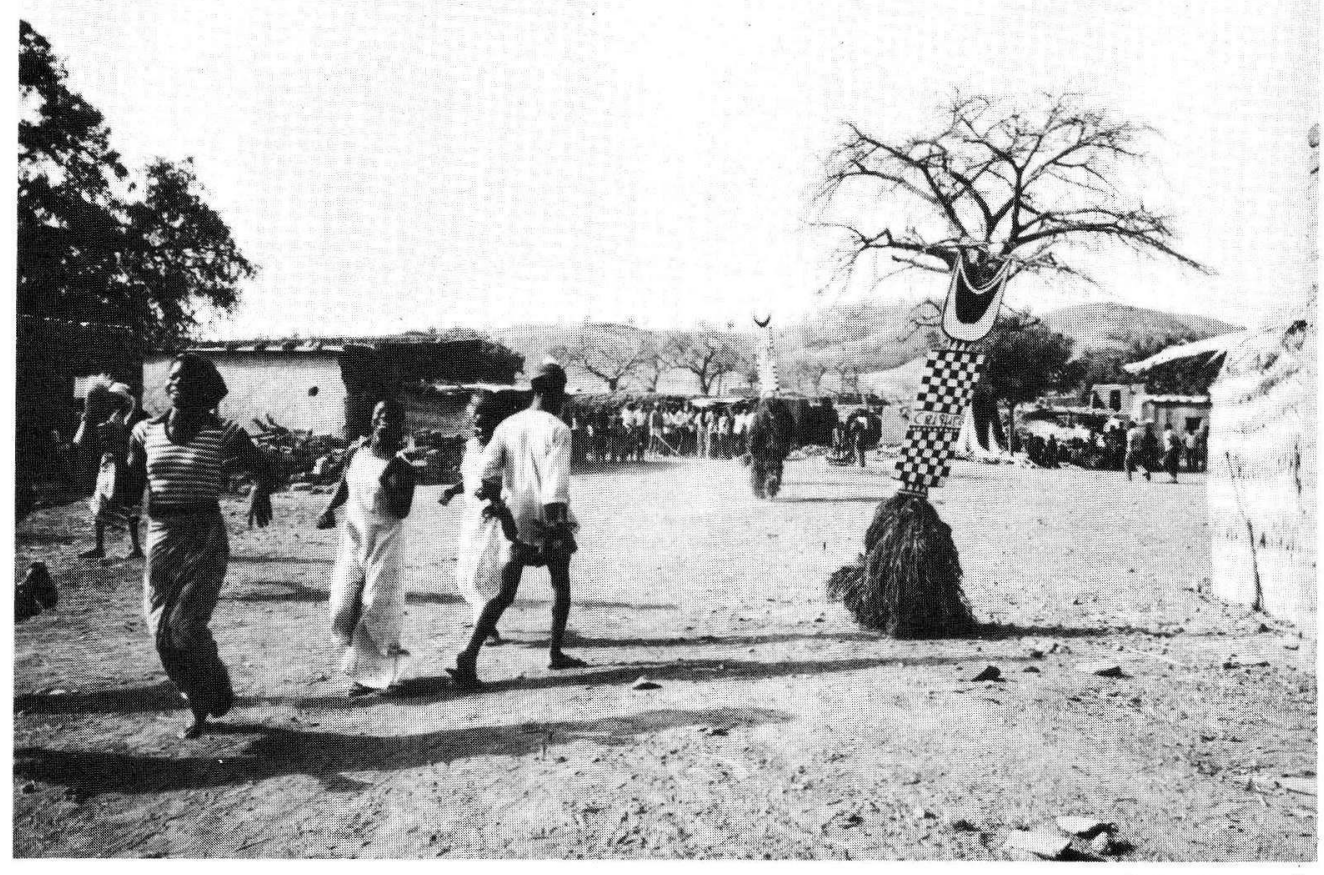

(cr. Coquet)

Fig. 7. Sortie de masques pour marquer $1 \mathrm{a}$ fin de la période d'initiation. La figuration en damier est la même que celle des couvertures tissées nunu. Village de Boni. 


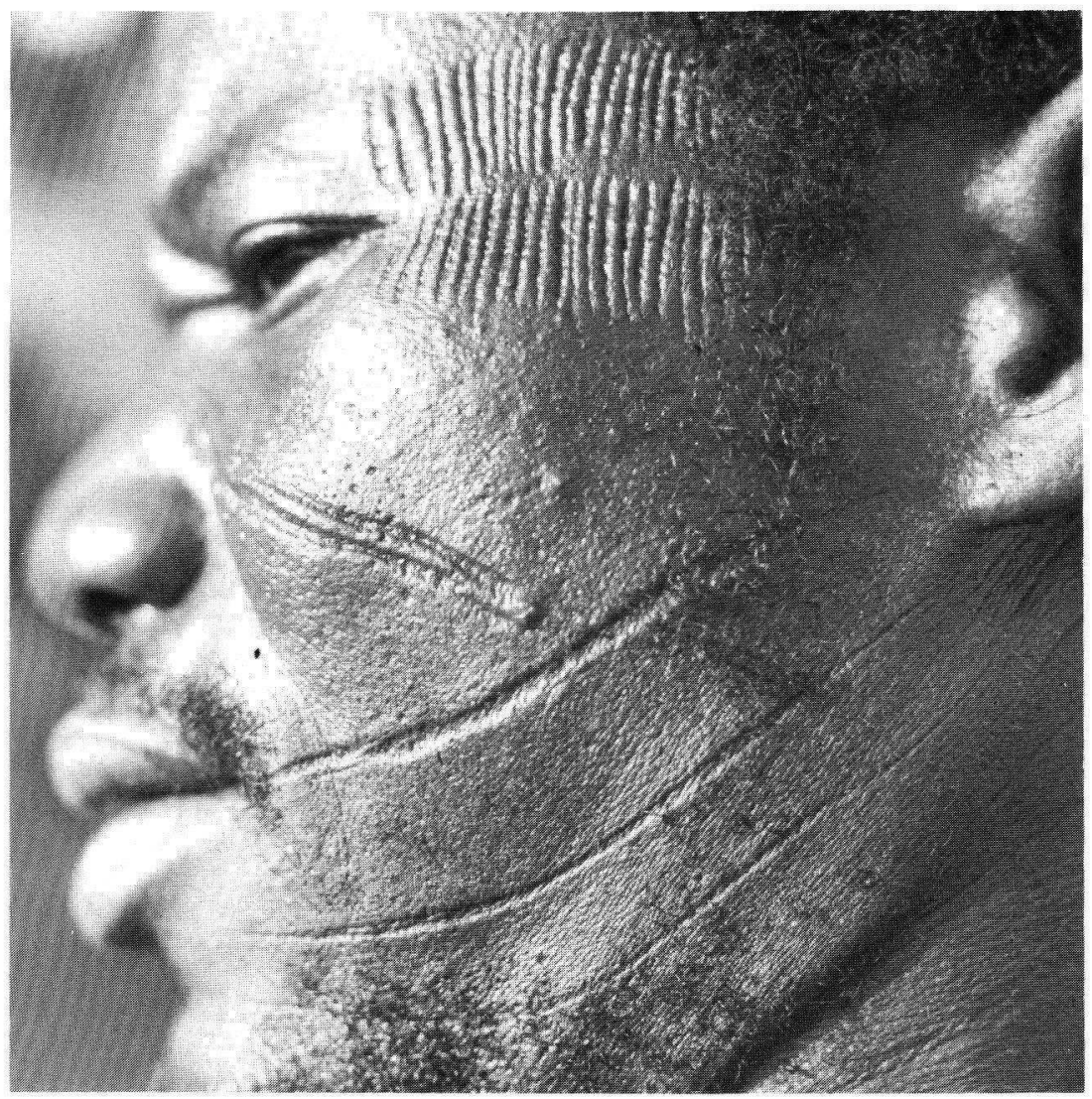

(ct. Coquet)

Fig. 8. Exemple de scarification dite de la cithare, cienhun, sur la tempe. 